www.nature.com/jhg

\title{
Estimation of carrier frequencies of six autosomal- recessive Mendelian disorders in the Korean population
}

\begin{abstract}
Min-Jung Song ${ }^{1}$, Seung-Tae Lee ${ }^{1}$, Mi-Kyung Lee ${ }^{2}$, Yongick Ji ${ }^{3}$, Jong-Won Kim ${ }^{1}$ and Chang-Seok Ki ${ }^{1}$
Although many studies have been performed to identify mutations in Korean patients with various autosomal-recessive Mendelian disorders (AR-MDs), little is known about the carrier frequencies of AR-MDs in the Korean population. Twenty common mutations from six AR-MDs, including Wilson disease (WD), non-syndromic hearing loss (NSHL), glycogen storage disease type la (GSD la), phenylketonuria (PKU), congenital hypothyroidism (CH), and congenital lipoid adrenal hyperplasia (CLAH) were selected to screen for based on previous studies. A total of $\mathbf{3 0 5 7}$ Koreans were genotyped by matrix-assisted laser desorption/ionization time-of-flight mass spectrometry followed by confirmation using the Sanger sequencing. We found 201 and 8 carriers with either one or two mutations in different genes, respectively, yielding a total carrier frequency of 1 in 15 (6.7\%). Of the six AR-MDs, NSHL has the highest carrier frequency followed by WD, CH, CLAH, GSD la, and PKU. As carrier screening tests are becoming prevalent and the number of mutations known and tested is rising, a priori data on the carrier frequencies in different ethnic groups is mandatory to plan a population screening program and to estimate its efficiency. In light of this, the present results can be used as a basis to establish a screening policy for common AR-MRs in the Korean population.
\end{abstract} Journal of Human Genetics (2012) 57, 139-144; doi:10.1038/jhg.2011.144; published online 15 December 2011

Keywords: autosomal-recessive disorder; carrier frequency; genetic screening; Korean

\section{INTRODUCTION}

Mendelian disorders are diseases that show a distinctive inheritance pattern due to mutations of a specific gene. ${ }^{1}$ In general, most Mendelian disorders are serious diseases that manifest at an early age, and for which there are few effective treatment options available. Thus, many efforts have been dedicated toward reducing the incidence of these diseases in many countries. In particular, diseases such as Tay-Sachs disease, cystic fibrosis and sickle cell anemia, which have a high prevalence among Ashkenazi Jews and ethnic groups from the Mediterranean region, as well as among European Caucasians and those from the United States, have been the focus of preventative efforts using various approaches, such as the detection of carriers of these disorders. ${ }^{2,3}$ Disorders that are suitable to population screening are usually autosomal-recessive Mendelian disorders (AR-MDs), but are sometimes X-linked Mendelian disorders. These diseases have the following features. First, they occur at different frequencies in various races. Second, although the frequencies of the disorders themselves are low, the frequencies of carriers are fairly high. Third, there are race-specific mutations. Finally, one or a few out of a large number of mutations present are dominant, occurring in $50-90 \%$ of carriers. ${ }^{1}$
Although the frequency of AR-MDs or XR-MRs is very low (1 in $10000-100000$ ), the frequency of carriers is relatively high (1 in $30-300) .{ }^{4}$ Moreover, patients have serious social and economic problems while carriers have no or subtle symptoms. However, according to recent research, carriers of mutations in certain genes that cause Mendelian disorders may have symptoms that emerge in old age. For example, a carrier of fragile $\mathrm{X}$ syndrome may develop fragile $\mathrm{X}$-associated tremor/ataxia syndrome after the age of 50 years, ${ }^{5}$ and it has been shown that carriers of Gaucher disease are highly likely to develop Parkinson's disease. ${ }^{6}$ Appropriate genetic counseling for patients and their family members can help them to understand their personal risk and the risk of other family members and to learn about testing, prevention strategies and surveillance.

In recent decades, the genes responsible for many of these disorders have been identified. Moreover, mutation analyses have revealed mutant alleles predominant in the Korean population. ${ }^{7-11}$ However, the carrier frequency and mutation spectrum of only a limited number of genes underlying these Mendelian diseases have been published. In the present study, we genotyped 3057 Korean individuals by highthroughput multiplex analysis on a Sequenom MassARRAY matrixassisted laser desorption/ionization time-of-flight (MALDI-TOF)

\footnotetext{
${ }^{1}$ Department of Laboratory Medicine and Genetics, Samsung Medical Center, Sungkyunkwan University School of Medicine, Seoul, Korea; ${ }^{2}$ Department of Laboratory Medicine, Chung-Ang University College of Medicine, Seoul, Korea and ${ }^{3}$ Center for Genome Research, Samsung Biomedical Research Institute, Sungkyunkwan University School of Medicine, Seoul, Korea

Correspondence: Dr C-S Ki, Department of Laboratory Medicine and Genetics, Samsung Medical Center, Sungkyunkwan University School of Medicine, 81 Irwon-ro, Gangnam-gu, Seoul 135-710, Korea.

E-mail: changski@skku.edu
}

Received 15 August 2011; revised 4 November 2011; accepted 25 November 2011; published online 15 December 2011 
mass spectrometry (MS) system (Sequenom, Inc., San Diego, CA, USA). Our aim in this study was to evaluate the carrier frequencies of relatively common AR-MDs in the Korean population, and to determine the distribution of common mutations involved in these diseases to provide a solid basis for the establishment of a screening policy for genetic testing.

\section{MATERIALS AND METHODS}

\section{Study populations}

The study population consisted of 3057 unrelated Koreans, ages 15-69 years (1529 males and 1528 females), who were recruited from the Health Promotion Center of a single institution between April 2007 and August 2010. Peripheral blood was collected from all subjects after obtaining informed consent, and this study was approved by the Institutional Review Board.

\section{Selection of study diseases and target mutations}

Six different diseases including Wilson disease (WD, MIM no. 277900), nonsyndromic hearing loss (NSHL), glycogen storage disease type Ia (GSD Ia, MIM no. 232200), phenylketonuria (PKU, MIM no. 261600), congenital hypothyroidism (CH, MIM no. 275200), and congenital lipoid adrenal hyperplasia (CLAH, MIM no. 201710) and 20 common mutations of each disease were selected arbitrarily for the study by literature review and analysis of patients in a Korean population. Selection criteria of study diseases is as follows; (1) relatively common AR-MRs in the Korean population ( $>1$ in 30000 ), or (2) although the incidence is low, carrier detection has the advantage of disease monitoring and timely therapeutic intervention for patients, and (3) the mutation spectrum data are available for Korean patients (Table 1).

\section{DNA preparation and MS}

Genomic DNA was isolated from peripheral blood leukocytes using the QIAamp DNA Blood Maxi kit (QIAGEN GmbH, Hilden, Germany). After storage at $-80^{\circ} \mathrm{C}$ for $1-4$ years, $10 \mathrm{ng}$ of genomic DNA was used as a template in the primary PCRs.

The targeted sequences were uploaded using MassArray Assay Design software v3.1.2.5 (Sequenom), to capture an approximately 80-120 bp region surrounding the targeted mutation (Supplementary Table S1). Extension primers that query the chosen mutation were designed to terminate at the base immediately adjacent to the targeted mutation (Supplementary Table S2).

Table 1 Study diseases and target mutations in the present study

\begin{tabular}{|c|c|c|}
\hline Disease & Gene & $\begin{array}{l}\text { Targeted } \\
\text { mutation }\end{array}$ \\
\hline \multirow[t]{7}{*}{ Wilson disease } & ATP7B & R778L \\
\hline & & A874V \\
\hline & & T1029। \\
\hline & & G1035V \\
\hline & & L1083F \\
\hline & & G1186S \\
\hline & & N1270s \\
\hline \multirow[t]{3}{*}{ Non-syndromic hearing loss } & GJB2 & V37I \\
\hline & & c.235delC \\
\hline & SLC26A4 & H723R \\
\hline \multirow[t]{2}{*}{ Glycogen storage disease type la } & G6PC & G122D \\
\hline & & c. $648 \mathrm{G}>\mathrm{T}$ \\
\hline \multirow[t]{6}{*}{ Phenylketonuria } & $P A H$ & IVS4-1G >A \\
\hline & & Y204C \\
\hline & & $\mathrm{R} 241 \mathrm{C}$ \\
\hline & & $\mathrm{R} 243 \mathrm{Q}$ \\
\hline & & T2781 \\
\hline & & Y356X \\
\hline Congenital hypothyroidism & TSHR & $\mathrm{R} 450 \mathrm{H}$ \\
\hline Congenital lipoid adrenal hyperplasia & StAR & Q258X \\
\hline
\end{tabular}

The MS method involves a multiplex primary PCR followed by an iPLEX reaction with a single primer of distinct mass that is specific for each genotype. Unextended primers demonstrate the absence of the mutation of interest, whereas specifically extended primers verify the presence and allow identification of specific genotypes. After laser deionization, the time of flight, which increases with $\mathrm{m} / \mathrm{z}$, is detected for each iPLEX primer.

\section{Primary PCR}

A PCR cocktail with a volume of $1820.2 \mu$ l. was prepared for 384 PCR reactions. The PCR cocktail comprised $875.5 \mu \mathrm{l}$ of water, $230.4 \mu \mathrm{l}$ of PCR Buffer, $184.3 \mu \mathrm{l}$ of $25 \mathrm{mM} \mathrm{MgCl}_{2}, 23.1 \mu \mathrm{l}$ of dNTP mix ( $25 \mathrm{~mm}$ each dNTP), $460.8 \mu \mathrm{l}$ of primer mix ( $500 \mathrm{~nm}$ each), and $46.1 \mu \mathrm{l}$ of PCR enzyme $\left(5 \mathrm{U}^{-1} \mathrm{l}^{-1}\right)$. One microliter of genomic DNA (5-10 $\mathrm{ngl}^{-1}$ ) was added to each well of a 384-well microtiter plate, and $4 \mu \mathrm{l}$ of the PCR cocktail was then dispensed into the wells. The plate was then cycled on a thermal cycler (Model T1 plus; Biometra, Goetingen, Germany) in a 384-well format in a volume of $5 \mu \mathrm{l}$ with the following profile: denaturation at $94^{\circ} \mathrm{C}$ for $4 \mathrm{~min}$, followed by 45 cycles of denaturation at $94{ }^{\circ} \mathrm{C}$ for $20 \mathrm{~s}$, annealing at $56^{\circ} \mathrm{C}$ for $30 \mathrm{~s}$, and extension at $72^{\circ} \mathrm{C}$ for $1 \mathrm{~min}$, followed by a final extension step at $72^{\circ} \mathrm{C}$ for $3 \mathrm{~min}$.

\section{iPLEX reaction}

Two microliter of iPLEX reaction mix was added to the dephosphorylated primary PCR reaction mix. Single base extension reactions were then cycled on a Biometra T1 plus using the following cycling profile: denaturation for $30 \mathrm{~s}$ at $94^{\circ} \mathrm{C}$, followed by 40 cycles of denaturation at $94^{\circ} \mathrm{C}$ for $5 \mathrm{~s}$, annealing at $52^{\circ} \mathrm{C}$ for $5 \mathrm{~s}$, and extension at $80^{\circ} \mathrm{C}$ for $5 \mathrm{~s}$, then an extension step at $72^{\circ} \mathrm{C}$ for $3 \mathrm{~min}$, and a final hold at $4{ }^{\circ} \mathrm{C}$. The reaction products were spotted onto a 384-spot SpectroCHIP with a MassARRAY Nanodispenser (Sequenom).

\section{MALDI-TOF MS analysis}

Samples were analyzed on a MassARRAY Analyzer Compact (Sequenom), which processed each 384-well chip in approximately $30 \mathrm{~min}$. Detailed information on peak heights for each assay and the probability value for each call, based on signal-to-noise ratios and peak probability statistics, were reviewed for each individual sample. In this system, probability was automatically calculated and parsed into three levels of stringency, resulting in conservative, moderate, and aggressive calls. Conservative calls resulted in the lowest rate of uncalled genotypes, whereas aggressive calls resulted in a higher error rate $(<1 \%)$. The Cluster Plot software module (Sequenom) was used to visualize atypical heterozygous and homozygous states.

\section{Validation of the MALDI-TOF MS method by Sanger sequencing} We validated a selective set of samples for each gene by Sanger sequencing using primer pairs that we designed for this study (Supplementary Table S3). A total of 209 samples that showed heterozygous mutations in MALDI-TOF MS analysis were selected for Sanger sequencing. Sanger sequencing was performed using an ABI Prism 3100xl Genetic Analyzer (Life Technologies, Carlsbad, CA, USA) and sequences were analyzed with reference to the wild-type sequence using the Sequencher program. We described sequence variation following the recommendations of the Human Genome Variation Society (www.hgvs.org/ mutnomen), designating the nucleotide $\mathrm{A}$ of the translation initiation codon ATG as +1 at the cDNA level and the corresponding methionine as +1 at the protein level.

\section{Statistical analyses}

We included a sufficient number of subjects to calculate the carrier frequencies for each of the six diseases that we investigated. ${ }^{12}$ The carrier frequency was defined as the number of heterozygotes of the studied population on the total number of individuals in the same population, which is approximately twice the allelic frequency. Carrier frequencies were calculated by direct counting and the carrier frequencies found in this study were compared with those reported for other populations using the $95 \%$ confidential interval. Estimated total carrier frequencies were deduced from the genotype frequencies. All statistical analyses were performed using SPSS statistical software (SPSS 11.0, http:// www.spss.com). 


\section{RESULTS}

In the present study, we examined a total of 3057 unrelated Korean individuals to identify carriers of disease-causing mutations that occur at a relatively high frequency in Korean patients with these diseases. We found that 201 individuals were carriers of one mutation and eight were carriers of two mutations in different genes related to development of the above-mentioned diseases. Therefore, the total estimated carrier frequency was 1 in $15(6.7 \%)$.

In WD, seven mutations in the ATP7B gene (NM_000053.2), namely, p.R778L, p.A874V, p.T1029I, p.G1035V, p.L1083F, p.G1186S, and p.N1270S, were screened for. Table 2 shows the frequency of WD mutated alleles found in our series of 3057 Koreans. Thirty-six individuals were carriers of one of the seven common mutations in the $A T P 7 B$ gene involved in WD. Thus, the carrier frequency of WDrelated mutations was 1 in 85 . The most common mutation was p.R778L, which was present in $44.4 \%$ of carriers, yielding a carrier frequency of 1 in 191. The p.N1270S and p.A874V mutations were found in $22.2 \%$ and $19.4 \%$ of carriers, respectively. For NSHL, 3,057 Korean individuals were screened for two mutations (c.235delC and p.V37I) found in the GJB2 gene (NM_004004.5) and one mutation (p.H723R) found in the SLC26A4 gene (NM_000441.1). Seventy-five individuals were carriers of one of the two mutations in the GJB2 gene (Table 3) and 41 were carriers of the p.H723R mutation in the
SLC26A4 gene (Table 4). The total carrier frequency for mutations in the GJB2 gene was 1 in 41 and in the SLC26A4 gene, 1 in 75. In GSD Ia, 17 subjects were carriers of one of the two known mutations (11 and 6 were carriers of the c.648G $>$ T and p.G122D mutations, respectively) in the G6PC gene (NM_000151). The carrier frequency was estimated to be 1 in 180 (Table 5). The most common mutation found among the Korean population was c.648G > T, which was found in $64.7 \%$ of the carriers studied, yielding a carrier frequency of 1 in 278. For PKU, we screened for six mutations in the PAH gene (NM_000277.1): c.442$1 \mathrm{G}>\mathrm{A}$ (IVS4-1G>A), p.Y204C, p.R241C, p.R243Q, p.T278I and p.Y356X. Of 3,057 Korean individuals, 11 were carriers of one of the six common mutations in the $P A H$ gene. This data indicate a carrier frequency of 1 in 278 (Table 5). The most common mutation, p.R243Q, was detected in $63.6 \%$ of the carriers, a carrier frequency of 1 in 437. No individuals had the p.Y204C or p.Y356X mutations. For $\mathrm{CH}$, we screened for the $\mathrm{p} . \mathrm{R} 450 \mathrm{H}$ mutation, because the mutation of the TSHR gene (NM_000369) accounts for 50\% of all mutant TSHR alleles in the Korean population; 20 subjects were found to be carriers of the p.R450H mutation. This equates to a carrier frequency of 1 in 153. For CLAH, 3057 individuals were screened for the p.Q258X mutation in the StAR gene (NM_000349.2) and 18 were found to be carriers of the mutation. The carrier frequency for CLAH in our study population is therefore 1 in 170 (Table 5).

Table 2 Wilson disease genotyping results in this study and reported carrier frequencies

\begin{tabular}{|c|c|c|c|c|c|}
\hline p.R778L & 39.2 & 16 & 1:191 & $1: 478$ & $1: 84$ \\
\hline p.A874V & 8.3 & 7 & $1: 437$ & $1: 239$ & $1: 250$ \\
\hline p.T1029l & 1.7 & 1 & $1: 3057$ & - & - \\
\hline p.G1186S & 1.6 & 0 & 0 & - & - \\
\hline p.N1270s & 6.7 & 8 & $1: 382$ & & $1: 250$ \\
\hline Total (\%) & 60.6 & 36 & $1: 85(1.18)$ & $1: 88(1.14)$ & $1: 50(2.00)$ \\
\hline $95 \% \mathrm{Cl}(\%)$ & & & $0.71-1.65$ & $0.19-2.09$ & $0.77-3.23$ \\
\hline
\end{tabular}

Abbreviations: $\mathrm{Cl}$, confidence interval; WD, Wilson disease.

Table 3 Reported carrier frequencies of the c.235deIC and p.V37I mutations in GJB2 gene in different populations

\begin{tabular}{|c|c|c|c|c|c|c|c|c|}
\hline \multirow[b]{2}{*}{ Population } & \multirow[b]{2}{*}{ Subjects tested } & \multicolumn{3}{|c|}{ c.235de/C } & \multicolumn{3}{|c|}{ V37I } & \multirow[b]{2}{*}{ References } \\
\hline & & No. of carriers & $\begin{array}{c}\text { Carrier frequency } \\
(\%)\end{array}$ & $95 \% \mathrm{Cl}$ & No. of carriers & $\begin{array}{c}\text { Carrier frequency } \\
(\%)\end{array}$ & $95 \% \mathrm{Cl}$ & \\
\hline \multirow[t]{3}{*}{ Korean } & 3057 & 27 & $1: 113(0.88)$ & $0.55-1.20$ & 48 & $1: 64(1.57)$ & $0.84-2.28$ & Current study \\
\hline & 2072 & 26 & $1: 80(1.25)$ & $0.77-1.73$ & 28 & $1: 74(1.35)$ & $0.85-1.85$ & Han et al. ${ }^{15}$ \\
\hline & 100 & 1 & $1: 100(1.00)$ & $0-2.95$ & - & - & - & Park et al. ${ }^{7}$ \\
\hline \multirow[t]{2}{*}{ Japanese } & 96 & 2 & $1: 48(2.08)$ & $0-4.93$ & 2 & $1: 48(2.08)$ & $0-4.93$ & Abe et $a .^{22}$ \\
\hline & 147 & 2 & $1: 74(1.35)$ & $0-3.21$ & 2 & $1: 74(1.35)$ & $0-3.21$ & Ohtsuka et al. ${ }^{23}$ \\
\hline \multirow[t]{2}{*}{ Chinese } & 368 & 5 & $1: 74(1.35)$ & $0.17-2.53$ & - & - & - & Dai et al. ${ }^{25}$ \\
\hline & 50 & 1 & $1: 50(2.00)$ & $0-5.88$ & 3 & $1: 17(5.88)$ & $0-12.4$ & Shi et al. ${ }^{24}$ \\
\hline \multirow[t]{3}{*}{ Caucasian } & 3516 & - & - & - & 30 & $1: 117(0.85)$ & $0.55-1.15$ & Roux et al. (2004) \\
\hline & 1047 & - & - & - & 9 & $1: 116(0.86)$ & $0.30-1.42$ & Pollak et al. (2007) \\
\hline & 116 & - & - & - & 1 & $1: 116(0.86)$ & $0-2.54$ & Marlin et al. (2001) \\
\hline
\end{tabular}

Abbreviation: $\mathrm{Cl}$, confidence interval. 
Table 4 Reported carrier frequencies of the H723R mutation in SLC26A4 gene

\begin{tabular}{|c|c|c|c|c|c|}
\hline Study & Population & No. screened & No. of carriers & Carrier frequency (\%) & $95 \% \mathrm{Cl}$ \\
\hline Park et al. ${ }^{8}$ & Korean & 120 & 2 & $1: 60(1.67)$ & $0-3.96$ \\
\hline
\end{tabular}

Abbreviation: $\mathrm{Cl}$, confidence interval.

Table 5 Disease incidence and detected carrier frequency in this study

\begin{tabular}{|c|c|c|c|c|c|c|}
\hline Disease & Incidence & $\begin{array}{c}\text { No. of carriers } \\
\text { detected } \\
(n=3057)\end{array}$ & $\begin{array}{c}\text { Carrier frequency } \\
\text { of related } \\
\text { mutations (\%) }\end{array}$ & $\begin{array}{c}95 \% \mathrm{Cl} \\
(\%)\end{array}$ & $\begin{array}{c}\text { Estimated total } \\
\text { carrier frequency of } \\
\text { each disorder }\end{array}$ & $95 \% \mathrm{Cl}(\%)$ \\
\hline WD & $1: 30000$ & 36 & $1: 85(1.18)$ & $0.71-1.65$ & $1: 51(1.95)$ & $1.46-2.44$ \\
\hline NSHL & $1: 1000$ & 116 & $1: 27(3.80)$ & $3.12-4.48$ & - & - \\
\hline GJB2-related & & 75 & $1: 41(2.45)$ & $1.91-3.00$ & & \\
\hline SLC26A4-related & & 41 & $1: 75(1.34)$ & $0.94-1.74$ & & \\
\hline GSD la & $1: 100000$ & 17 & $1: 180(0.56)$ & $0.28-0.82$ & $1: 161(0.62)$ & $0.48-0.76$ \\
\hline PKU & $1: 41000$ & 11 & $1: 278(0.36)$ & $0.15-0.57$ & $1: 118(0.85)$ & $0.53-1.17$ \\
\hline $\mathrm{CH}$ & $1: 3500$ & 20 & $1: 153(0.65)$ & $0.37-0.93$ & - & - \\
\hline $\mathrm{CLAH}$ & $1: 300000$ & 18 & $1: 170(0.59)$ & $0.32-0.86$ & $1: 135(0.74)$ & $0.44-1.04$ \\
\hline
\end{tabular}

Abbreviations: $\mathrm{CH}$, congenital hypothyroidism; $\mathrm{Cl}$, confidence interval; CLAH, congenital lipoid adrenal hyperplasia; GSD la, glycogen storage disease type la; M:F, male:female;

NSHL, non-syndromic hearing loss; PKU, phenylketonuria; WD, Wilson disease.

\section{DISCUSSIONS}

In the present study, we generated and analyzed a database containing 61140 genotyping assay results from 3057 nonselected Korean individuals. We determined the number of carriers with common diseasecausative mutations of WD, NSHL, GSD Ia, PKU, CH, and CLAH, which are Mendelian disorders with a higher frequency in the Korean population than other populations. ${ }^{8,10,11,13}$ We found that 201 individuals out of 3,057 were carriers of a mutation in one of the genes and eight individuals were carriers of two mutations in different genes underlying the development of the above-mentioned diseases. On the basis of the number of individual carriers, the total carrier frequency in our study population was 1 in $15(6.7 \%)$ of the individuals assessed. There was no significant difference between males and females with regard to carrier frequencies for the different disease. Our results update the previously reported carrier frequencies for WD $(1 \text { in } 88)^{14}$ and NSHL due to the GJB2- or SLC26A4-related mutations ( 1 in 38 and 1 in 60 , respectively) ${ }^{8,15}$ In addition, this is the first report of carrier frequencies for GSD Ia, PKU, CH due to the TSHR-related mutations, and CLAH in the Korean population by molecular genetic analysis.

WD is a disorder of copper metabolism caused by mutations in the $A T P 7 B$. In the present study, the carrier frequency of WD-related mutations was 1 in 85 , similar to that previously reported ( 1 in $88-$ $90) ;{ }^{14,16}$ however, this carrier frequency is lower than that reported by Park et al. ${ }^{11}$ Given that the seven mutations that we investigated represent $60.6 \%$ of the genetic variation in Korean WD patients, ${ }^{17}$ we presume that the actual carrier frequency of WD in the Korean is approximately 1 in 51 .

Congenital hearing loss is a common disorder and about $50 \%$ of the causes are of genetic origin. ${ }^{18}$ Genetic deafness is highly heterogeneous, with more than 100 genes predicted to cause this disorder. ${ }^{19}$ In spite of this large heterogeneity, mutations in the GJB2 and SLC26A4 account for up to $50 \%{ }^{20}$ and $10 \%$ of hereditary HL in diverse populations, ${ }^{21}$ respectively. In the study, the total frequency of carriers with mutations in the GJB2 was 1 in 41, similar to that previously reported in the Korean population. ${ }^{7,15}$ As shown in Table 3, this is slightly lower than the carrier frequencies reported for Korea's neighboring countries, Japan and China; these differences might be because of the small number of subjects tested in those countries. ${ }^{22-25}$ The frequency of carriers with mutations in the SLC26A4 was 1 in 75, similar to the carrier frequencies reported previously in the Korean population $^{8}$ and Japanese population ${ }^{21}$ (Table 4). Given that the one mutation that we investigated represents $45.5 \%$ of the genetic variation in Korean hearing-loss patients with the SLC26A4 mutations, ${ }^{8}$ we presume that the carrier frequency of the SLC26A4-related mutations in the Korean population is 1 in 34 .

GSD Ia is the most common type of GSD I and caused by a deficiency of glucose-6-phosphatase activity. Two mutations in the G6PC, c.648G $>$ T and p.G122D, are responsible for GSD Ia in approximately $90 \%$ of Korean patients with this disease. ${ }^{9}$ The carrier frequency detected in this study is 1 in 180 . Given that the two mutations that we investigated represent $90 \%$ of the genetic variation in Korean GSD Ia patients, the actual carrier frequency of GSD Ia is approximately 1 in 161 in the Korean population (Table 5).

PKU is an inborn error of metabolism caused by a deficiency of phenylalanine hydroxylase. The mutation profile of the $P A H$ is not restricted to any one region, but is spread throughout the structural domains and shows enormous diversity. The carrier frequency of the mutations involved in PKU found in the study ( 1 in 278) is lower than that previously reported in the Japanese population (1 in 200) based on newborn screening. ${ }^{26}$ Given that the six mutations investigated represent $42.3 \%$ of the genetic variation in Korean PKU patients, ${ }^{10}$ we presume that the carrier frequency of PKU is approximately 1 in 118 (Table 5). Thus, we can infer that the carrier frequency of PKU in the Koreans is higher than that in the Japanese.

The majority of $\mathrm{CH}$ cases $(75-80 \%)$ are caused by defects in the development of the thyroid gland (thyroid dysgenesis), whereas abnormalities in thyroid hormone synthesis (dyshormonogenesis) account for a minor proportion of cases (15-20\%). ${ }^{27}$ Although the 
pathogenesis of $\mathrm{CH}$ remains largely unknown, mutations in several genes (for example, TSHR, FOXE1, PAX8, TPO and TG) have been implicated. ${ }^{28}$ Of these genes, the TSHR has been reported to be one of the major disease-causative genes in many populations. ${ }^{29,30}$ A single mutation in the TSHR, p.R $450 \mathrm{H}$, accounts for more than $50 \%$ of TSHR mutations in Korean patients and more than $75 \%$ in Japanese patients. ${ }^{30}$ The carrier frequency detected in the study was 1 in 153 (Table 5), similar to the mono-allelic mutation carrier frequency ( 1 in 172) previously reported in the Japanese population (based on HardyWeinberg equilibrium). Considering that we only examined the carrier frequency of the p.R450H mutation in TSHR, the total carrier frequency of $\mathrm{CH}$ in the Korean population is likely to be higher than that in the Japanese population. As there is significant genetic heterogeneity of $\mathrm{CH}$, however, a population-based genetic screening of $T S H R$ for the detection of $\mathrm{CH}$ carriers might produce limited results. Therefore, further genetic studies dealing with other causative genes will be necessary to reach for the total genetic landscape of $\mathrm{CH}$ and more appropriate genetic counseling.

CLAH is a disorder of steroid biosynthesis and the most severe form of congenital adrenal hyperplasia. Interestingly, most cases occur in individuals from Korea, Japan and Palestinian Arabs. Furthermore, the p.Q258X mutation is most commonly found in both Korean and Japanese patients with CLAH. ${ }^{13,32}$ The carrier frequency detected in the study was 1 in 170 (Table 5). Given that the one mutation that we investigated accounts for more than $80 \%$ of the genetic variation in Korean CLAH patients with StAR mutations, the actual carrier frequency of CLAH in the Korean population is likely to be approximately 1 in 135 .

The results of our present study indicate a high carrier frequency for WD and NSHL in the Korean population (greater than 1 in 60). The current recommendations by the Israel Society of Human Genetics suggest that genetic disorders with frequencies greater than 1 in 60 should be screened for, ${ }^{4}$ indicating that WD and NSHL should be screened for in Korea. In addition, GSD Ia, PKU, CH and CLAH are serious childhood diseases. Some of these diseases are lethal and others affect development, and sufferers are unable to live independently. Moreover, the carriers of $\mathrm{CH}$ and WD usually show subclinical disease manifestations related to decreased enzyme activity. ${ }^{33}$ Therefore, we highly recommend molecular genetic prenatal screening of Korean individuals to identify relevant mutations. In addition, it is important to screen for carriers in the Korean population to identify couples that are heterozygous for fatal genetic diseases, in order to reduce the incidence of these diseases and prevent homozygosity for these disorders. Information about carrier frequencies and common mutations in each disease should be made available to the public and to professionals involved in genetic counseling for carrier screening. In contrast, mutations that are frequent among other ethnic populations but rare among Koreans should not be included in carrier screening protocols. Our results provide a fundamental basis for the establishment of a screening policy for genetic testing in the Korean population.

The ability to diagnose and predict the risk for fatal childhood diseases is an excellent advancement. However, inexpensive and highly sensitive tests with low false-positive results are needed for genetic screening. At present, the majority of molecular genetic testing is not automated and the cost of testing remains one of the major obstacles. In this study, we analyzed the mutations with a high-throughput method, MALDI-TOF MS. Comparative analysis of 209 samples showed excellent agreement between the results obtained by direct sequencing and MALDI-TOF MS. Moreover, mutation typing by the MALDI-TOF method has a major advantage over other established methods. MS analysis uses at least an order of magnitude less DNA $(<10 \mathrm{ng}$ ), with much higher throughput and greater sensitivity and accuracy. ${ }^{34,35}$ The inclusion of a primer extension step after PCR also increases precision and accommodates DNA samples of poorer quality. Therefore, the MALDI-TOF approach could provide greater precision with fewer assays than direct sequencing and useful to examine allele frequencies in a large number of samples.

In summary, this is the first large-scale study to report the carrier frequencies of several genetic disorders simultaneously in the Korean population. As screening tests are becoming prevalent and the number of mutations known and tested is rising, it is desirable to increase the efficiency of testing and to lower the costs. Elucidating the spectrum and frequency of each mutation is essential to unravel the genetic basis of Mendelian diseases in the Korean population and to facilitate prenatal screening, carrier detection and counseling.

\section{ACKNOWLEDGEMENTS}

This study was supported by a grant from the Samsung Biomedical Research Institute (\#SBRI C-B0-206-1).

1 Antonarakis, S. E. \& Beckmann, J. S. Mendelian disorders deserve more attention. Nat. Rev. Genet. 7, 277-282 (2006).

2 Frumkin, A. \& Zlotogora, J. Genetic screening for reproductive purposes at school: is it a good strategy? Am. J. Med. Genet. A. 146A, 264-269 (2008).

3 Zlotogora, J. Population programs for the detection of couples at risk for severe monogenic genetic diseases. Hum. Genet. 126, 247-253 (2009).

4 Fares, F., Badarneh, K., Abosaleh, M., Harari-Shaham, A., Diukman, R. \& David, M. Carrier frequency of autosomal-recessive disorders in the Ashkenazi Jewish population: should the rationale for mutation choice for screening be reevaluated? Prenat. Diagn. 28, 236-241 (2008)

5 Leehey, M. A. Fragile X-associated tremor/ataxia syndrome: clinical phenotype, diagnosis, and treatment. J. Investig. Med. 57, 830-836 (2009).

6 DePaolo, J., Goker-Alpan, O., Samaddar, T., Lopez, G. \& Sidransky, E. The association between mutations in the lysosomal protein glucocerebrosidase and parkinsonism. Mov. Disord. 24, 1571-1578 (2009).

7 Park, H. J., Hahn, S. H., Chun, Y. M., Park, K. \& Kim, H. N. Connexin26 mutations associated with nonsyndromic hearing loss. Laryngoscope 110, 1535-1538 (2000).

8 Park, H. J., Shaukat, S., Liu, X. Z., Hahn, S. H., Naz, S., Ghosh, M. et al. Origins and frequencies of SLC26A4 (PDS) mutations in east and south Asians: global implications for the epidemiology of deafness. J. Med. Genet. 40, 242-248 (2003).

9 Ki, C. S., Han, S. H., Kim, H. J., Lee, S. G., Kim, E. J., Kim, J. W. et al. Mutation spectrum of the glucose-6-phosphatase gene and its implication in molecular diagnosis of Korean patients with glycogen storage disease type la. Clin. Genet. 65, 487-489 (2004).

10 Lee, Y. W., Lee, D. H., Kim, N. D., Lee, S. T., Ahn, J. Y., Choi, T. Y. et al. Mutation analysis of PAH gene and characterization of a recurrent deletion mutation in Korean patients with phenylketonuria. Exp. Mol. Med. 40, 533-540 (2008).

11 Park, H. D., Ki, C. S., Lee, S. Y. \& Kim, J. W. Carrier frequency of the R778L, A874V, and N1270S mutations in the ATP7B gene in a Korean population. Clin. Genet. 75, 405-407 (2009).

12 Collins, J. S. \& Schwartz, C. E. Detecting polymorphisms and mutations in candidate genes. Am. J. Hum. Genet. 71, 1251-1252 (2002)

13 Bose, H. S., Sugawara, T., Strauss, J. F. III \& Miller, W. L. The pathophysiology and genetics of congenital lipoid adrenal hyperplasia. N. Engl. J. Med. 335, 1870-1878 (1996).

14 Kim, G. H., Yang, J. Y., Park, J. Y., Lee, J. J., Kim, J. H. \& Yoo, H. W. Estimation of Wilson's disease incidence and carrier frequency in the Korean population by screening ATP7B major mutations in newborn filter papers using the SYBR green intercalator method based on the amplification refractory mutation system. Genet. Test. 12, 395399 (2008).

15 Han, S. H., Park, H. J., Kang, E. J., Ryu, J. S., Lee, A., Yang, Y. H. et al. Carrier frequency of GJB2 (connexin-26) mutations causing inherited deafness in the Korean population. J. Hum. Genet. 53, 1022-1028 (2008).

16 Scheinberg, I. \& Sternlieb, I. Wilson's disease In: Smith LH Jr (ed) Major Problems in Internal Medicine 23, 12-13 (1984).

17 Park, S., Park, J. Y., Kim, G. H., Choi, J. H., Kim, K. M., Kim, J. B. et al. Identification of novel ATP7B gene mutations and their functional roles in Korean patients with Wilson disease. Hum. Mutat. 28, 1108-1113 (2007).

18 Petit, C., Levilliers, J. \& Hardelin, J. P. Molecular genetics of hearing loss. Annu. Rev. Genet. 35, 589-646 (2001).

19 Steel, K. P. \& Kros, C. J. A genetic approach to understanding auditory function. Nat. Genet. 27, 143-149 (2001). 
20 Smith, R. J., Bale, J. F. Jr. \& White, K. R. Sensorineural hearing loss in children. Lancet 365, 879-890 (2005).

21 Tsukamoto, K. Suzuki, H., Harada, D., Namba, A., Abe, S. \& Usami, S. Distribution and frequencies of PDS (SLC26A4) mutations in Pendred syndrome and nonsyndromic hearing loss associated with enlarged vestibular aqueduct: a unique spectrum of mutations in Japanese. Eur. J. Hum. Genet. 11, 916-922 (2003).

22 Abe, S., Usami, S., Shinkawa, H., Kelley, P. M. \& Kimberling, W. J. Prevalent connexin 26 gene (GJB2) mutations in Japanese. J. Med. Genet. 37, 41-43 (2000).

23 Ohtsuka, A., Yuge, I., Kimura, S., Namba, A., Abe, S., Van Laer, L. et al. GJB2 deafness gene shows a specific spectrum of mutations in Japan, including a frequent founder mutation. Hum. Genet. 112, 329-333 (2003).

24 Shi, G. Z., Gong, L. X., Xu, X. H., Nie, W. Y., Lin, Q. \& Qi, Y. S. GJB2 gene mutations in newborns with non-syndromic hearing impairment in Northern China. Hear. Res. 197, 19-23 (2004).

25 Dai, P., Yu, F., Han, B., Yuan, Y., Li, Q., Wang, G. et al. The prevalence of the 235deIC GJB2 mutation in a Chinese deaf population. Genet. Med. 9, 283-289 (2007).

26 Aoki, K. \& Wada, Y. Outcome of the patients detected by newborn screening in Japan. Acta. Paediatr. Jpn. 30, 429-434 (1988).

27 Jain, V., Agarwal, R., Deorari, A. K. \& Paul, V. K. Congenital hypothyroidism. Indian. J. Pediatr. 75, 363-367 (2008).

28 Park, S. M. \& Chatterjee, V. K. Genetics of congenital hypothyroidism. J. Med. Genet. 42, 379-389 (2005).
29 Cangul, H., Morgan, N. V., Forman, J. R., Saglam, H., Aycan, Z., Yakut, T. et al. Novel TSHR mutations in consanguineous families with congenital nongoitrous hypothyroidism. Clin. Endocrinol. (Oxf). 73, 671-677.

30 Narumi, S., Muroya, K., Abe, Y., Yasui, M., Asakura, Y., Adachi, M. et al. TSHR mutations as a cause of congenital hypothyroidism in Japan: a population-based genetic epidemiology study. J. Clin. Endocrinol. Metab. 94, 1317-1323 (2009).

31 Tee, M. K., Lin, D., Sugawara, T., Holt, J. A., Guiguen, Y., Buckingham, B. et al. T $\rightarrow$ A transversion $11 \mathrm{bp}$ from a splice acceptor site in the human gene for steroidogenic acute regulatory protein causes congenital lipoid adrenal hyperplasia. Hum. Mol. Genet. 4, 2299-2305 (1995).

32 Yoo, H. W. \& Kim, G. H. Molecular and clinical characterization of Korean patients with congenital lipoid adrenal hyperplasia. J. Pediatr. Endocrinol. Metab. 11, 707-711 (1998).

33 Persani, L., Calebiro, D., Cordella, D., Weber, G., Gelmini, G., Libri, D. et al. Genetics and phenomics of hypothyroidism due to TSH resistance. Mol. Cell. Endocrinol. 322, 72-82.

34 Farkas, D. H., Miltgen, N. E., Stoerker, J., van den Boom, D., Highsmith, W. E., Cagasan, L. et al. The suitability of matrix assisted laser desorption/ionization time of flight mass spectrometry in a laboratory developed test using cystic fibrosis carrier screening as a model. J. Mol. Diagn. 12, 611-619.

35 Houtchens, K. A., Nichols, R. J., Ladner, M. B., Boal, H. E., Sollars, C., Geraghty, D. E. et al. High-throughput killer cell immunoglobulin-like receptor genotyping by MALDITOF mass spectrometry with discovery of novel alleles. Immunogenetics 59, 525-537 (2007).

Supplementary Information accompanies the paper on Journal of Human Genetics website (http://www.nature.com/jhg) 